\title{
ON STOCHASTIC PREDICTIONS OF FAILURE PROCESSES UNDER POPULATION HETEROGENEITY
}

\author{
JI HWAN CHA,* Ewha Womans University
}

\begin{abstract}
In reliability a number of failure processes for repairable items are described by point processes, depending on the types of repairs being performed on failures of items. In this paper we describe the failure processes of repairable items from heterogeneous populations and study the stochastic predictions of future processes which utilize the failure/repair history. Two types of repair processes, perfect and minimal repair processes, will be considered. The results will be derived under a general stochastic formulation/setting. Applications of the obtained results to many different areas will be discussed and, specifically, some reliability applications will be illustrated in detail.
\end{abstract}

Keywords: Failure process; repairable item; heterogeneous population; stochastic prediction; process history; stochastic intensity

2010 Mathematics Subject Classification: Primary 60K10

Secondary $62 \mathrm{P} 30$

\section{Introduction}

Recently, there has been a rapidly increasing literature concerning modeling and analysis of recurrent events, with a wide range of applications, including reliability analysis of repairable items.

Traditionally, the literature on repairable items is concerned with modeling failure processes, with point process theory being the main tool. The most commonly used models for the failure process of repairable items are renewal processes and nonhomogeneous Poisson processes (NHPPs), including the homogeneous Poisson process (HPP) as a special case in both models. These point processes are closely related to the types of repair actions performed for the items on their failures. The NHPP is often used to model repairable items that are subject to a minimal repair strategy, whereas the renewal process is adopted to describe the failure process of items subject to a perfect repair strategy (see Aven and Jensen (1999)).

While the basic and fundamental theory on point processes developed for such models might often be sufficient for simple reliability analysis, the need for advanced studies on more complex and practical problems has recently been emerging, aiming at significant contributions to relevant areas. The main interest of the problems in this stream has been focused on the 'stochastic prediction' of random recurrent events.

One of the most important emerging issues in this area is the study of the stochastic behavior of random recurrent events in a random time interval. For instance, in Esary et al. (1973), Grandell (1997), Badía and Sanggüessa (2008), and Badía (2011), the authors obtained suitable conditions under which the reliability properties of a random time $T$ are inherited by $N(T)$,

Received 30 August 2011; revision received 30 November 2012.

* Postal address: Department of Statistics, Ewha Womans University, Seoul, 120-750, Korea.

Email address: jhcha@ewha.ac.kr 
where $\{N(t): t \geq 0\}$ is a stochastic counting process independent of $T$. Through the studies on this issue, one may 'predict' the stochastic behavior of the random number of events in an independent random interval.

Another important issue might be to 'predict' future failure processes based on the failure/repair history of repairable items. In many cases, the 'prior information' about the items under operation might not be sufficient and, thus, as a result, we may have a certain degree of 'uncertainty'. In these cases, the operational history of the repairable items generally conveys additional information about the reliability characteristic of the items under operation and, combined with the prior information that we already have, it may produce updated 'posterior information'. This could make it possible to predict the future behavior of the failure process under consideration. In spite of the practical importance of this issue, to the best of the author's knowledge, little effort has been paid to this topic in reliability.

Frequently, the 'uncertainty' exists in the information about the population from which our items are being selected. This may happen especially when we are dealing with heterogeneous populations. For example, in some situations, items may come from one of $k$ subpopulations, each with a distinct lifetime distribution. A reliability engineer, for instance, might have a component that was manufactured in one of $k$ facilities, but is not certain which facility the item came from.

In this paper we describe the failure processes of repairable items from heterogeneous populations and study the stochastic predictions of the future processes of items. Two kinds of repair models, perfect and minimal repair models, will be considered and, accordingly, the renewal process and NHPP will be our main concerns. For the purpose of a convenient description of the model, we formulate the problem in the context of reliability modeling. However, the approaches and obtained results could straightforwardly be reformulated and extended within the general point process context, which might entail general contributions of this work to various relevant areas.

This paper is organized as follows. In Section 2, the basic mathematical setup for the model formulation will be built. Then, considering the perfect repair process, the stochastic predictions of future failure processes will be investigated. In Section 3, the point process for minimal repair will be studied and an application to reliability will be illustrated. In Section 4 , various potential applications of the obtained results will be discussed and future research topics will also be suggested. Finally, some concluding remarks will be given.

\section{Point process for perfect repair}

In this section we consider a perfect repair strategy for items under operation. If the items under operation and perfect repair processes are from a homogeneous population, then the failure process of the repairable item follows an ordinary renewal process. In this situation, the future failure process from time $t$ depends only on the age of the item in use at time $t$ (i.e. the time measured backwards from $t$ to the last renewal point) and it can be straightforwardly predicted. However, the situation becomes dramatically different for heterogeneous settings. We start the formulation of our problem with an introductory example, which has only an explanatory purpose.

Example 1. Consider a stock of $k$ substocks (substock 1, substock 2, ., substock $k$ ) of identical items, which are manufactured by $k$ different manufacturers. Suppose that the lifetime of items in each substock has an absolutely continuous distribution and, thus, its distribution can be characterized by the corresponding distribution function $F(t, i)$ and failure 
rate function $\lambda(t, i), i=1,2, \ldots, k$. Assume that at time $t=0$ one item is chosen from a substock which was randomly selected in accordance with a discrete distribution $\pi(i), i=$ $1,2, \ldots, k$. This item is put into operation and the operating item is 'perfectly' repaired on its failure. Here, by 'perfect repair' we mean that the state of the item after the repair is restored to an 'as-good-as-new' condition. For instance, if the initial item is chosen from substock $i$ then this type of repair implies that the distribution function of the perfectly repaired item is given by $F(t, i)$. In practice, this type of repair can be realized by a series of repair actions which would bring each part of the item to a 'brand new' state (i.e. by a complete overhaul), or by replacing the failed item with an identical new item 'from the same substock'.

Based on this simple consideration, we now formulate our problem under the more general setting of a continuous mixing model. However, the formulation can be easily modified for a simpler case (e.g. the discrete mixing model illustrated in Example 1) when it is more suitable. Let $T$ be a lifetime random variable (RV) of an item with cumulative distribution function (CDF) $F(t)(\bar{F}(t) \equiv 1-F(t))$. Assume that $F(t)$ is absolutely continuous and, therefore, the probability density function (PDF) $f(t)=F^{\prime}(t)$ exists. It is well known that the failure rate function is then defined as

$$
\lambda(t) \equiv \lim _{\Delta t \rightarrow 0} \frac{\mathbb{P}[t<T \leq t+\Delta t \mid T>t]}{\Delta t}=\frac{f(t)}{\bar{F}(t)}, \quad t \geq 0 .
$$

Based on (1), the failure rate function for items from heterogeneous populations can also be formally defined as follows (see, e.g. Finkelstein (2008, Chapter 6), (2009)). Assume that $F(t)$ is indexed by an $\mathrm{RV} Z, \mathbb{P}(T \leq t \mid Z=z) \equiv F(t, z)$, and that the corresponding $\operatorname{PDF} f(t, z)$ exists. Then the corresponding failure rate $\lambda(t, z)$ is defined by $\lambda(t, z)=f(t, z) / \bar{F}(t, z)$, where $\bar{F}(t, z) \equiv 1-F(t, z)$. Let $Z$ be a nonnegative RV with support in $[a, b], 0 \leq a<b \leq \infty$, and PDF $\pi(z)$. Without loss of generality, let $a=0$ and $b=\infty$. The above setting leads naturally to considering mixtures of distributions, i.e.

$$
F_{m}(t)=\int_{0}^{\infty} F(t, z) \pi(z) \mathrm{d} z
$$

whereas the mixture (population) failure rate, in accordance with the definition, is

$$
\lambda_{m}(t)=\frac{\int_{0}^{\infty} f(t, z) \pi(z) \mathrm{d} z}{\int_{0}^{\infty} \bar{F}(t, z) \pi(z) \mathrm{d} z}=\int_{0}^{\infty} \lambda(t, z) \pi(z \mid t) \mathrm{d} z,
$$

where $\pi(z \mid t)$ is the conditional PDF (on condition that $T>t$ ) defined by

$$
\pi(z \mid t) \equiv \pi(z) \frac{\bar{F}(t, z)}{\int_{0}^{\infty} \bar{F}(t, w) \pi(w) \mathrm{d} w} .
$$

Under this mixing structure, as in the motivating example (Example 1), we consider the perfect repair process (i.e. items are perfectly repaired on failures) with the frailty variable $Z=z$ of the initial item 'preserved'. Obviously, this point process is not a renewal process as the interarrival times are dependent. However, even in this case, stochastic measures of the point process can be described as the point process, given $Z=z$, follows a renewal process. Therefore, this point process can be called a conditional renewal process.

Suppose that this point process is started at time $t=-s$, but that the process is not observed before time $t=0$. As usual, let $N(t), t>0$, be the number of failures in the 
time interval $(0, t]$. We start the observation on the point process at time $t=0$ and observe it in the time interval $[0, t)$. For a simple formulation of the problem, we assume in the following that the time point $t=0$ is not a renewal point, but the generalization of the following discussions to this special case is straightforward. Then our objective is to predict the future failure process based on the observation of the history of the process in $[0, t)$. For simplicity, we omit the starting point ' $-s$ ' in all the notation to be defined, but it should be noted that the starting point is 'implicitly' contained in the corresponding notation.

Let $\mathscr{H}_{t} \equiv\{N(u), 0 \leq u<t\}$ be the history in $[0, t)$, and let $h_{t}$ be the realization of the corresponding history. Observe that $\mathscr{H}_{t}$ can be completely defined in terms of $N(t-)$ and the sequential arrival failure points $0<S_{1}<S_{2}<\cdots<S_{N(t-)}<t$ in $[0, t)$, where $S_{i}$ is the time from 0 until the $i$ th failure in $[0, t)$. Then, equivalently, $\mathscr{H}_{t}$ can be defined in terms of $N(t-)$ and the interarrival times $T_{1}, T_{2}, \ldots, T_{N(t-)}: \mathscr{H}_{t}=\left\{N(t-), T_{1}, T_{2}, \ldots, T_{N(t-)}\right\}$, where $T_{1} \equiv S_{1}$, $T_{i} \equiv S_{i}-S_{i-1}, i=2,3, \ldots, N(t-)$.

A convenient way to describe the mathematics of orderly point processes is to use the concept of the stochastic intensity (the intensity process) $\lambda_{t}, t \geq 0$ (see Aven and Jensen (1999, pp. 5456) and Finkelstein (2008, pp. 70-76)). In our discussions below, as our main objective is to predict the future failure process based on the observation of the history in $[0, t)$, we define the general stochastic intensity

$$
\lambda_{x \mid \mathscr{H}_{t}} \equiv \lim _{\Delta t \rightarrow 0} \frac{\mathbb{P}\left[N(x, x+\Delta t)=1 \mid \mathscr{H}_{t}\right]}{\Delta t}=\lim _{\Delta t \rightarrow 0} \frac{\mathbb{E}\left[N(x, x+\Delta t) \mid \mathscr{H}_{t}\right]}{\Delta t}, \quad x \geq t,
$$

where $N\left(t_{1}, t_{2}\right), t_{1}<t_{2}$, represents the number of failures in $\left[t_{1}, t_{2}\right)$. Then the above general stochastic intensity has the heuristic interpretation

$$
\lambda_{x \mid \mathscr{H}_{t}} \mathrm{~d} x=\mathbb{E}\left[\mathrm{d} N(x) \mid \mathscr{H}_{t}\right], \quad x \geq t .
$$

Observe that the general stochastic intensity in (4) with $s=0$ and $x=t$ reduces to the ordinary stochastic intensity $\lambda_{t}, t \geq 0$, in Aven and Jensen (1999, p. 54) and Finkelstein (2008, p. 71). In the following, by convention, the notation $\lambda_{x \mid h_{t}}$ is defined as

$$
\lambda_{x \mid h_{t}} \equiv \lim _{\Delta t \rightarrow 0} \frac{\mathbb{P}\left[N(x, x+\Delta t)=1 \mid \mathscr{H}_{t}=h_{t}\right]}{\Delta t} .
$$

For convenience, define $N_{t}(u) \equiv N(t+u)-N(t), u>0$, which is the number of failures during $u$ units of time from $t$, and $T_{t}$ as the time from $t$ until the next failure. In addition, we define $A(w)$ and $Y(w)$ as the 'age' and the forward recurrent time of the item in use at time $w$, respectively. Then, clearly, $T_{t}$ defined above is equal to $Y(t)$. We are now ready to describe the stochastic measures for the future process $\left\{N_{t}(u), u \geq 0\right\}$ in the following theorem. In the following, by convention, $\prod_{i=2}^{n}(\cdot) \equiv 1$ for $n=1$. Furthermore, let $H(t)$ and $G(t)$ be CDFs and $h(t)$ and $g(t)$ be the corresponding PDFs, respectively. Then $H^{(r)}$ is the $r$-fold convolution of the CDF $H(t)$ and $h^{(r)}$ is the corresponding PDF. Also, the convolution of $H$ and $G$ is denoted by the conventional notation $H * G$ and the corresponding PDF is denoted by $h * g$. Note that, when the frailty variable $Z=z$ is given, these convolutions will be defined with fixed $z$. For example, $H^{(2)}(t, z) \equiv \int_{0}^{t} H(t-u, z) h(u, z) \mathrm{d} u$ and $H * G(t, z) \equiv \int_{0}^{t} H(t-u, z) g(u, z) \mathrm{d} u$.

Theorem 1. Let $w_{z}(r) \equiv \sum_{k=1}^{\infty} f^{(k)}(r, z)$. Under the perfect repair process, given $\mathscr{H}_{t}$, the stochastic measures for the process $\left\{N_{t}(u), u \geq 0\right\}$ are given as follows. 
Case I: $h_{t}=\left\{N(t-)=n, T_{1}=t_{1}, T_{2}=t_{2}, \ldots, T_{n}=t_{n}\right\}, n \geq 1$. Let $s_{n} \equiv \sum_{i=1}^{n} t_{i}$, $f_{D 1}(w, z) \equiv \lambda\left(t-s_{n}+w, z\right) \exp \left\{-\int_{0}^{w} \lambda\left(t-s_{n}+v, z\right) \mathrm{d} v\right\}$, and $w_{z}^{D 1}(r) \equiv \sum_{k=1}^{\infty} f_{D 1} *$ $f^{(k-1)}(r, z)$. Then

(i) $\lambda_{t+u \mid h_{t}}=\int_{0}^{\infty}\left\{\lambda\left(t-s_{n}+u, z\right) \exp \left\{-\int_{0}^{u} \lambda\left(t-s_{n}+v, z\right) \mathrm{d} v\right\}\right.$

$$
\left.+\int_{0}^{u} \lambda(r, z) \cdot(1-F(r, z)) w_{z}^{D 1}(u-r) \mathrm{d} r\right\} \pi_{t}(z) \mathrm{d} z
$$

(ii) $\mathbb{P}\left[T_{t} \geq x \mid \mathscr{H}_{t}=h_{t}\right]=\int_{0}^{\infty} \exp \left\{-\int_{0}^{x} \lambda\left(t-s_{n}+v, z\right) \mathrm{d} v\right\} \pi_{t}(z) \mathrm{d} z$,

(iii) $\mathbb{P}\left[N_{t}(u)=k \mid \mathscr{H}_{t}=h_{t}\right]=\int_{0}^{\infty}\left[F_{T_{t} \mid h_{t}} * F^{(k-1)}(u, z)-F_{T_{t} \mid h_{t}} * F^{(k)}(u, z)\right] \pi_{t}(z) \mathrm{d} z$,

(iv) $\mathbb{E}\left[N_{t}(u) \mid \mathscr{H}_{t}=h_{t}\right]=\int_{0}^{\infty}\left[\sum_{k=1}^{\infty} F_{T_{t} \mid h_{t}} * F^{(k-1)}(u, z)\right] \pi_{t}(z) \mathrm{d} z$,

where

$$
\begin{gathered}
\pi_{t}(z) \equiv \frac{f_{Y(0)}\left(t_{1}, z\right) \prod_{i=2}^{n}\left(\lambda\left(t_{i}, z\right) \exp \left\{-\int_{0}^{t_{i}} \lambda(w, z) \mathrm{d} w\right\}\right) \exp \left\{-\int_{0}^{t-\sum_{i=1}^{n} t_{i}} \lambda(w, z) \mathrm{d} w\right\} \pi(z)}{\int_{0}^{\infty} f_{Y(0)}\left(t_{1}, v\right) \prod_{i=2}^{n}\left(\lambda\left(t_{i}, v\right) \exp \left\{-\int_{0}^{t_{i}} \lambda(w, v) \mathrm{d} w\right\}\right) \exp \left\{-\int_{0}^{\left.t-\sum_{i=1}^{n}{ }^{t_{i}} \lambda(w, v) \mathrm{d} w\right\} \pi(v) \mathrm{d} v},\right.} \\
f_{Y(0)}(x, z) \equiv f(s+x, z)+\int_{0}^{s} f(v+x, z) w_{z}(s-v) \mathrm{d} v
\end{gathered}
$$

and

$$
F_{T_{t} \mid h_{t}}(x, z) \equiv 1-\exp \left\{-\int_{0}^{x} \lambda\left(t-s_{n}+v, z\right) \mathrm{d} v\right\} .
$$

Case II: $h_{t}=\{N(t-)=0\}$. Let $f_{D 2, a}(w, z) \equiv \lambda(a+t+w, z) \exp \left\{-\int_{0}^{w} \lambda(a+t+v, z) \mathrm{d} v\right\}$ and $w_{z, a}^{D 2}(r) \equiv \sum_{k=1}^{\infty} f_{D 2, a} * f^{(k-1)}(r, z)$. Then

(i) $\lambda_{t+u \mid h_{t}}=\int_{0}^{\infty} \int_{0}^{s}\left\{\lambda(a+t+u, z) \exp \left\{-\int_{0}^{u} \lambda(a+t+v, z) \mathrm{d} v\right\}\right.$

$$
\begin{gathered}
\left.+\int_{0}^{u} \lambda(r, z)(1-F(r, z)) w_{z, a}^{D 2}(u-r) \mathrm{d} r\right\} \phi_{t}^{1}(a, z) \mathrm{d} a \mathrm{~d} z \\
+\int_{0}^{\infty}\left\{\lambda(s+t+u, z) \exp \left\{-\int_{0}^{u} \lambda(s+t+v, z) \mathrm{d} v\right\}\right. \\
\left.+\int_{0}^{u} \lambda(r, z)(1-F(r, z)) w_{z, s}^{D 2}(u-r) \mathrm{d} r\right\} \phi_{t}^{2}(s, z) \mathrm{d} z,
\end{gathered}
$$

(ii) $\mathbb{P}\left[T_{t} \geq x \mid \mathscr{H}_{t}=h_{t}\right]=\int_{0}^{\infty} \int_{0}^{s} \exp \left\{-\int_{0}^{x} \lambda(a+t+v, z) \mathrm{d} v\right\} \phi_{t}^{1}(a, z) \mathrm{d} a \mathrm{~d} z$

$$
+\int_{0}^{\infty} \exp \left\{-\int_{0}^{x} \lambda(s+t+v, z) \mathrm{d} v\right\} \phi_{t}^{2}(s, z) \mathrm{d} z
$$

(iii) $\mathbb{P}\left[N_{t}(u)=k \mid \mathscr{H}_{t}=h_{t}\right]$

$$
\begin{aligned}
= & \int_{0}^{\infty} \int_{0}^{s}\left\{F_{T_{t} \mid h_{t}, a} * F^{(k-1)}(u, z)-F_{T_{t} \mid h_{t}, a} * F^{(k)}(u, z)\right\} \phi_{t}^{1}(a, z) \mathrm{d} a \mathrm{~d} z \\
& +\int_{0}^{\infty}\left\{F_{T_{t} \mid h_{t}, s} * F^{(k-1)}(u, z)-F_{T_{t} \mid h_{t}, s} * F^{(k)}(u, z)\right\} \phi_{t}^{2}(s, z) \mathrm{d} z,
\end{aligned}
$$


(iv) $\mathbb{E}\left[N_{t}(u) \mid \mathscr{H}_{t}=h_{t}\right]=\int_{0}^{\infty} \int_{0}^{s}\left\{\sum_{k=1}^{\infty} F_{T_{t} \mid h_{t}, a} * F^{(k-1)}(u, z)\right\} \phi_{t}^{1}(a, z) \mathrm{d} a \mathrm{~d} z$

$$
+\int_{0}^{\infty}\left\{\sum_{k=1}^{\infty} F_{T_{t} \mid h_{t}, s} * F^{(k-1)}(u, z)\right\} \phi_{t}^{2}(s, z) \mathrm{d} z,
$$

where

$$
\begin{aligned}
\phi_{t}^{1}(a, z) \equiv & \frac{\exp \left\{-\int_{0}^{t} \lambda(a+w, z) \mathrm{d} w\right\} f_{A(0)}(a, z) \pi(z)}{\xi(t, s)} \\
f_{A(0)}(a, z) \equiv & (1-F(a, z)) w_{z}(s-a), \quad 0 \leq a<s \\
\xi(t, s) \equiv & \int_{0}^{\infty} \int_{0}^{s} \exp \left\{-\int_{0}^{t} \lambda(b+w, v) \mathrm{d} w\right\} f_{A(0)}(b, v) \pi(v) \mathrm{d} b \mathrm{~d} v \\
& +\int_{0}^{\infty} \exp \left\{-\int_{0}^{t} \lambda(s+w, v) \mathrm{d} w\right\} \exp \left\{-\int_{0}^{s} \lambda(w, v) \mathrm{d} w\right\} \pi(v) \mathrm{d} v, \\
\phi_{t}^{2}(s, z) \equiv & \frac{\exp \left\{-\int_{0}^{t} \lambda(s+w, z) \mathrm{d} w\right\} \exp \left\{-\int_{0}^{s} \lambda(w, z) \mathrm{d} w\right\} \pi(z)}{\xi(t, s)},
\end{aligned}
$$

and

$$
F_{T_{t} \mid h_{t}, a}(x, z) \equiv 1-\exp \left\{-\int_{0}^{x} \lambda(a+t+v, z) \mathrm{d} v\right\} .
$$

Proof. We first consider case I, that is, $h_{t}=\left\{N(t-)=n, T_{1}=t_{1}, T_{2}=t_{2}, \ldots\right.$, $\left.T_{n}=t_{n}\right\}, n \geq 1$.

(i) Observe that the general stochastic intensity in (4) can now be specified as

$$
\begin{aligned}
& \lambda_{x \mid \mathcal{H}_{t}}=\lim _{\Delta t \rightarrow 0} \frac{\mathbb{E}\left[\mathbb{P}\left[N(x, x+\Delta t)=1 \mid \mathscr{H}_{t}, Z\right]\right]}{\Delta t} \\
& =\mathbb{E}\left[\lim _{\Delta t \rightarrow 0} \frac{\mathbb{P}\left[N(x, x+\Delta t)=1 \mid \mathscr{H}_{t}, Z\right]}{\Delta t}\right]
\end{aligned}
$$

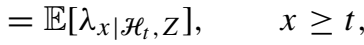

where all the expectations in the equations in (5) are with respect to the 'conditional distribution' of $\left(Z \mid \mathscr{H}_{t}\right)$, and

$$
\lambda_{x \mid \mathscr{H}_{t}, Z} \equiv \lim _{\Delta t \rightarrow 0} \frac{\mathbb{P}\left[N(x, x+\Delta t)=1 \mid \mathscr{H}_{t}, Z\right]}{\Delta t}, \quad x \geq t .
$$

Here, for a fixed $x, \lambda_{x \mid \mathscr{H}_{t}, Z}$ is the function of $\left(\mathscr{H}_{t}, Z\right)$ whose value when $\mathscr{H}_{t}=h_{t}$ and $Z=z$ is

$$
\lim _{\Delta t \rightarrow 0} \frac{\mathbb{P}\left[N(x, x+\Delta t)=1 \mid \mathscr{H}_{t}=h_{t}, Z=z\right]}{\Delta t}, \quad x \geq t,
$$

which will be denoted by $\lambda_{x \mid h_{t}, z}$.

We now derive $\lambda_{t+u \mid \mathscr{H}_{t}, Z}$; we will then take its expectation with respect to the conditional distribution $Z \mid \mathscr{H}_{t}$ according to (5). Observe that

$$
\lambda_{t+u \mid h_{t}, z}=\lim _{\Delta t \rightarrow 0} \frac{\mathbb{P}\left[N(t+u, t+u+\Delta t)=1 \mid \mathscr{H}_{t}=h_{t}, Z=z\right]}{\Delta t} .
$$


Here, $\mathbb{P}\left[N(t+u, t+u+\Delta t)=1 \mid \mathscr{H}_{t}=h_{t}, Z=z\right]$ can be obtained by

$$
\begin{aligned}
& \mathbb{P}\left[N(t+u, t+u+\Delta t)=1 \mid \mathscr{H}_{t}=h_{t}, Z=z\right] \\
& \quad=\mathbb{E}\left[\mathbb{P}\left[N(t+u, t+u+\Delta t)=1 \mid \mathscr{H}_{t}=h_{t}, Z=z, A(t+u)\right]\right],
\end{aligned}
$$

where the expectation is with respect to the conditional distribution of $\left(A(t+u) \mid \mathscr{H}_{t}=h_{t}\right.$, $Z=z$ ). Note that, given $\mathscr{H}_{t}=h_{t}$ and $Z=z$, the counting process 'which starts from time $t$ ' is the 'delayed renewal process' with the first interarrival time distribution (PDF)

$$
f_{D 1}(w, z) \equiv \lambda\left(t-s_{n}+w, z\right) \exp \left\{-\int_{0}^{w} \lambda\left(t-s_{n}+v, z\right) \mathrm{d} v\right\},
$$

and the common remaining interarrival times distribution (PDF) $f(w, z)$. Then, given $\mathscr{H}_{t}=h_{t}$ and $Z=z$, the 'age' of the item at time $t+u, A(t+u)$, is equal to $t-s_{n}+u$ with probability $\exp \left\{-\int_{0}^{u} \lambda\left(t-s_{n}+v, z\right) \mathrm{d} v\right\}$ (discrete part), and the PDF of the continuous part of the distribution of $\left(A(t+u) \mid \mathscr{H}_{t}=h_{t}, Z=z\right)$ is

$$
(1-F(r, z)) w_{z}^{D 1}(u-r), \quad 0 \leq r<u,
$$

where $w_{z}^{D 1}(r) \equiv \sum_{k=1}^{\infty} f_{D 1} * f^{(k-1)}(r, z)$ (see Cox (1962, p. 61)). Now, in accordance with (6),

$$
\begin{aligned}
\mathbb{P}[N(t+ & \left.u, t+u+\Delta t)=1 \mid \mathscr{H}_{t}=h_{t}, Z=z\right] \\
= & \mathbb{P}\left[N(t+u, t+u+\Delta t)=1 \mid \mathscr{H}_{t}=h_{t}, Z=z, A(t+u)=t-s_{n}+u\right] \\
& \times \exp \left\{-\int_{0}^{u} \lambda\left(t-s_{n}+v, z\right) \mathrm{d} v\right\} \\
& +\int_{0}^{u} \mathbb{P}\left[N(t+u, t+u+\Delta t)=1 \mid \mathscr{H}_{t}=h_{t}, Z=z, A(t+u)=r\right] \\
& \times(1-F(r, z)) w_{z}^{D 1}(u-r) \mathrm{d} r .
\end{aligned}
$$

Note that

$$
\begin{gathered}
\mathbb{P}\left[N(t+u, t+u+\Delta t)=1 \mid \mathscr{H}_{t}=h_{t}, Z=z, A(t+u)=w\right] \\
=1-\exp \left\{-\int_{0}^{\Delta t} \lambda(w+v, z) \mathrm{d} v\right\}+o(\Delta t),
\end{gathered}
$$

and, thus,

$$
\lim _{\Delta t \rightarrow 0} \frac{\mathbb{P}\left[N(t+u, t+u+\Delta t)=1 \mid \mathscr{H}_{t}=h_{t}, Z=z, A(t+u)=w\right]}{\Delta t}=\lambda(w, z) .
$$

Therefore, we have

$$
\begin{aligned}
\lambda_{t+u \mid h_{t}, z}= & \lambda\left(t-s_{n}+u, z\right) \exp \left\{-\int_{0}^{u} \lambda\left(t-s_{n}+v, z\right) \mathrm{d} v\right\} \\
& +\int_{0}^{u} \lambda(r, z)(1-F(r, z)) w_{z}^{D 1}(u-r) \mathrm{d} r .
\end{aligned}
$$

Now the conditional distribution $Z \mid \mathscr{H}_{t}$ will be constructed. The conditional distribution of the history $\mathscr{H}_{t}=h_{t}$ given $Z=z$ is

$$
f_{Y(0)}\left(t_{1}, z\right) \prod_{i=2}^{n}\left(\lambda\left(t_{i}, z\right) \exp \left\{-\int_{0}^{t_{i}} \lambda(w, z) \mathrm{d} w\right\}\right) \exp \left\{-\int_{0}^{t-\sum_{i=1}^{n} t_{i}} \lambda(w, z) \mathrm{d} w\right\}
$$


where $f_{Y(0)}(x, z)$ is the PDF of the forward recurrence time (the conditional PDF given $Z=z$ ) of an item operating at time 0 under the corresponding repair process, started at time ' $-s$ ', and is given by (see, e.g. Rausand and Høyland (2004, Section 7.3.6))

$$
f_{Y(0)}(x, z)=f(s+x, z)+\int_{0}^{s} f(v+x, z) w_{z}(s-v) \mathrm{d} v, \quad 0 \leq x<\infty,
$$

with $w_{z}(r) \equiv \sum_{k=1}^{\infty} f^{(k)}(r, z)$. Then the conditional PDF of $Z$ given $\mathscr{H}_{t}=h_{t}$, which is denoted by $\pi_{t}(z)$, is given by

$$
\pi_{t}(z)=\frac{f_{Y(0)}\left(t_{1}, z\right) \prod_{i=2}^{n}\left(\lambda\left(t_{i}, z\right) \exp \left\{-\int_{0}^{t_{i}} \lambda(w, z) \mathrm{d} w\right\}\right) \exp \left\{-\int_{0}^{t-\sum_{i=1}^{n} t_{i}} \lambda(w, z) \mathrm{d} w\right\} \pi(z)}{\int_{0}^{\infty} f_{Y(0)}\left(t_{1}, v\right) \prod_{i=2}^{n}\left(\lambda\left(t_{i}, v\right) \exp \left\{-\int_{0}^{t_{i}} \lambda(w, v) \mathrm{d} w\right\}\right) \exp \left\{-\int_{0}^{t-\sum_{i=1}^{n}{ }^{t_{i}}} \lambda(w, v) \mathrm{d} w\right\} \pi(v) \mathrm{d} v} .
$$

Finally, in accordance with (5), $\lambda_{t+u \mid h_{t}}$ can be obtained as

$$
\lambda_{t+u \mid h_{t}}=\int_{0}^{\infty} \lambda_{t+u \mid h_{t}, z} \pi_{t}(z) \mathrm{d} z
$$

(ii) By similar arguments to those given in (i), it is clear that

$$
\mathbb{P}\left[T_{t} \geq x \mid \mathscr{H}_{t}\right]=\mathbb{E}\left[\mathbb{P}\left[T_{t} \geq x \mid \mathscr{H}_{t}, Z\right]\right],
$$

where the expectation is with respect to the conditional distribution $Z \mid \mathscr{H}_{t}$. Obviously,

$$
\mathbb{P}\left[T_{t} \geq x \mid \mathscr{H}_{t}=h_{t}, Z=z\right]=\exp \left\{-\int_{0}^{x} \lambda\left(t-s_{n}+v, z\right) \mathrm{d} v\right\},
$$

and, therefore, the desired result is obtained.

(iii) Clearly, given $\mathscr{H}_{t}=h_{t}$ and $Z=z$, the process $\left\{N_{t}(u), u \geq 0\right\}$ is a delayed renewal process with the first interarrival time distribution

$$
F_{T_{t} \mid h_{t}}(x, z)=1-\exp \left\{-\int_{0}^{x} \lambda\left(t-s_{n}+v, z\right) \mathrm{d} v\right\} .
$$

Therefore, the result can now be obtained by constructing the corresponding conditional distribution and then taking an appropriate expectation.

(iv) The result is directly obtained from (iii).

Now we consider case II, i.e. $h_{t}=\{N(t-)=0\}$.

(i) When $h_{t}=\{N(t-)=0\}$, given the history, the age of an item operating at time $0, A(0)$, is still a random variable. In this case, similar to (5),

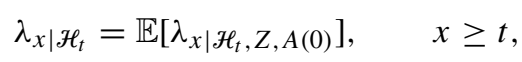

where the expectation is with respect to the joint conditional distribution $A(0), Z \mid \mathscr{H}_{t}$, and

$$
\lambda_{x \mid \mathscr{H}_{t}, Z, A(0)} \equiv \lim _{\Delta t \rightarrow 0} \frac{\mathbb{P}\left[N(x, x+\Delta t)=1 \mid \mathscr{H}_{t}, Z, A(0)\right]}{\Delta t}, \quad x \geq t .
$$

Here, the corresponding limit and the notation $\lambda_{x \mid h_{t}, z, a}$ are defined as in case I. Then

$$
\lambda_{t+u \mid h_{t}, z, a}=\lim _{\Delta t \rightarrow 0} \frac{\mathbb{P}\left[N(t+u, t+u+\Delta t)=1 \mid \mathscr{H}_{t}=h_{t}, Z=z, A(0)=a\right]}{\Delta t} .
$$


Here, $\mathbb{P}\left[N(t+u, t+u+\Delta t)=1 \mid \mathscr{H}_{t}=h_{t}, Z=z, A(0)=a\right]$ can be obtained by

$$
\begin{aligned}
& \mathbb{P}\left[N(t+u, t+u+\Delta t)=1 \mid \mathscr{H}_{t}=h_{t}, Z=z, A(0)=a\right] \\
& \quad=\mathbb{E}\left[\mathbb{P}\left[N(t+u, t+u+\Delta t)=1 \mid \mathscr{H}_{t}=h_{t}, Z=z, A(0)=a, A(t+u)\right]\right],
\end{aligned}
$$

where the expectation is with respect to the conditional distribution of $\left(A(t+u) \mid \mathscr{H}_{t}=h_{t}\right.$, $Z=z, A(0)=a)$. Now, given $\mathscr{H}_{t}=h_{t}, Z=z$, and $A(0)=a$, the counting process 'which starts from time $t$ ' is the 'delayed renewal process' with the first interarrival time distribution (PDF)

$$
f_{D 2, a}(w, z) \equiv \lambda(a+t+w, z) \exp \left\{-\int_{0}^{w} \lambda(a+t+v, z) \mathrm{d} v\right\},
$$

and the common remaining interarrival times distribution (PDF) $f(w, z)$. Then, given $\mathscr{H}_{t}=h_{t}$, $Z=z$, and $A(0)=a$, the 'age' of the item at time $t+u, A(t+u)$, is equal to $a+t+u$ with probability $\exp \left\{-\int_{0}^{u} \lambda(a+t+v, z) \mathrm{d} v\right\}$ (discrete part), and the PDF of the continuous part of the distribution of $\left(A(t+u) \mid \mathscr{H}_{t}=h_{t}, Z=z, A(0)=a\right)$ is

$$
(1-F(r, z)) w_{z, a}^{D 2}(u-r), \quad 0 \leq r<u,
$$

where $w_{z, a}^{D 2}(r) \equiv \sum_{k=1}^{\infty} f_{D 2, a} * f^{(k-1)}(r, z)$. Then, following a procedure similar to that given in case I(i), $\lambda_{t+u \mid h_{t}, z, a}$ can be obtained as

$$
\begin{aligned}
\lambda_{t+u \mid h_{t}, z, a}= & \lambda(a+t+u, z) \exp \left\{-\int_{0}^{u} \lambda(a+t+v, z) \mathrm{d} v\right\} \\
& +\int_{0}^{u} \lambda(r, z)(1-F(r, z)) w_{z, a}^{D 2}(u-r) \mathrm{d} r .
\end{aligned}
$$

Let $\phi_{t}(a, z)$ be the conditional joint PDF of $(A(0), Z)$ given $\mathscr{H}_{t}=h_{t}$. Observe that the conditional probability of the history $\mathscr{H}_{t}=h_{t}$ given $(A(0)=a, Z=z)$ is

$$
\exp \left\{-\int_{0}^{t} \lambda(a+w, z) \mathrm{d} w\right\}
$$

whereas the 'nonabsolutely continuous' joint distribution of $(A(0), Z)$, which has its marginal point mass at $a=s$, is given by

$$
\begin{gathered}
f_{A(0)}(a, z) \pi(z), \quad 0 \leq a<s, 0 \leq z<\infty, \\
\exp \left\{-\int_{0}^{s} \lambda(w, z) \mathrm{d} w\right\} \pi(z), \quad a=s, 0 \leq z<\infty,
\end{gathered}
$$

where $f_{A(0)}(a, z)$ is the PDF of the continuous part of the distribution $A(0) \mid Z=z$ and is given by $f_{A(0)}(a, z)=(1-F(a, z)) w_{z}(s-a), 0 \leq a<s$. Therefore, the nonabsolutely continuous conditional joint distribution of $(A(0), Z), \phi_{t}(a, z)$, can be constructed as

$$
\phi_{t}(a, z)= \begin{cases}\frac{\exp \left\{-\int_{0}^{t} \lambda(a+w, z) \mathrm{d} w\right\} f_{A(0)}(a, z) \pi(z)}{\xi(t, s)}, & 0 \leq a<s, 0 \leq z<\infty, \\ \frac{\exp \left\{-\int_{0}^{t} \lambda(s+w, z) \mathrm{d} w\right\} \exp \left\{-\int_{0}^{s} \lambda(w, z) \mathrm{d} w\right\} \pi(z)}{\xi(t, s)}, & a=s, 0 \leq z<\infty,\end{cases}
$$


where

$$
\begin{aligned}
\xi(t, s) \equiv & \int_{0}^{\infty} \int_{0}^{s} \exp \left\{-\int_{0}^{t} \lambda(b+w, v) \mathrm{d} w\right\} f_{A(0)}(b, v) \pi(v) \mathrm{d} b \mathrm{~d} v \\
& +\int_{0}^{\infty} \exp \left\{-\int_{0}^{t} \lambda(s+w, v) \mathrm{d} w\right\} \exp \left\{-\int_{0}^{s} \lambda(w, v) \mathrm{d} w\right\} \pi(v) \mathrm{d} v .
\end{aligned}
$$

Then, in accordance with (8),

$$
\begin{aligned}
\lambda_{t+u \mid h_{t}}= & \int_{0}^{\infty} \int_{0}^{s} \lambda_{t+u \mid h_{t}, z, a} \frac{\exp \left\{-\int_{0}^{t} \lambda(a+w, z) \mathrm{d} w\right\} f_{A(0)}(a, z) \pi(z)}{\xi(t, s)} \mathrm{d} a \mathrm{~d} z \\
& +\int_{0}^{\infty} \lambda_{t+u \mid h_{t}, z, s} \frac{\exp \left\{-\int_{0}^{t} \lambda(s+w, z) \mathrm{d} w\right\} \exp \left\{-\int_{0}^{s} \lambda(w, z) \mathrm{d} w\right\} \pi(z)}{\xi(t, s)} \mathrm{d} z .
\end{aligned}
$$

Results (ii), (iii), and (iv) can also be obtained following similar arguments to those given in case I, by first conditioning the corresponding measure on $\left(\mathscr{H}_{t}, A(0), Z\right)$ and then taking the expectation with respect to the conditional distribution of $\left(A(0), Z \mid \mathscr{H}_{t}\right)$.

Remark 1. (i) For recurrent events of natural disasters, such as earthquakes and river floods, the starting point of the process is generally given by ' $-\infty$ '. In this case, $f_{Y(0)}(x, z)$ is simplified to

$$
f_{Y(0)}(x, z)=\frac{1}{\int_{0}^{\infty} \bar{F}(t, z) \mathrm{d} t} \bar{F}(x, z), \quad 0 \leq x<\infty .
$$

Also, in this case, the nonabsolutely continuous distributions in case II become absolutely continuous distributions with

$$
f_{A(0)}(a, z)=\frac{1}{\int_{0}^{\infty} \bar{F}(t, z) \mathrm{d} t} \bar{F}(a, z), \quad 0 \leq a<\infty,
$$

and $s \equiv \infty$.

(ii) Observe that $\lambda_{m}(t)$ in (2) corresponds to $\lambda_{t+u \mid h_{t}}$ in case II(i) with $s=0, u=0$, and $A(0) \equiv 0$ (i.e. $A(0)$ has a degenerate distribution) as the given history $\{T>t\}$ in the former case corresponds to $h_{t}=\{N(t-)=0\}$. In this case, $\phi_{t}(a, z)$ in (10) reduces to the onedimensional distribution $\pi(z \mid t)$ in (3) and, accordingly, we have $\lambda_{m}(t)=\lambda_{t \mid h_{t}}$.

In the following example we consider the point process with interarrival times following (conditionally given $Z$ ) exponential distributions.

Example 2. Suppose that $F(t, z)$ is an exponential distribution with parameter $\lambda(t, z)=z \lambda$, and let $\pi(z)$ be an exponential PDF in $[0, \infty)$ with parameter $\theta$.

Case I: suppose that $h_{t}=\left\{N(t-)=n, T_{1}=t_{1}, T_{2}=t_{2}, \ldots, T_{n}=t_{n}\right\}$. Then, in this case,

$$
f_{Y(0)}(x, z)=z \lambda \exp \{-z \lambda x\}, \quad x \geq 0,
$$

which does not depend on the starting point $s$ and, accordingly, the stochastic measures are independent of $s$. Obviously, $\lambda_{t+u \mid h_{t}, z}$ in (7) is simply given by

$$
\lambda_{t+u \mid h_{t}, z}=z \lambda
$$


On the other hand,

$$
\begin{aligned}
\pi_{t}(z) & =\frac{(z \lambda)^{n} \exp \{-z \lambda t\} \theta \exp \{-\theta z\}}{\int_{0}^{\infty}(v \lambda)^{n} \exp \{-v \lambda t\} \theta \exp \{-\theta v\} \mathrm{d} v} \\
& =\frac{(\theta+\lambda t)^{n+1} z^{n} \exp \{-(\theta+\lambda t) z\}}{\Gamma(n+1)}, \quad 0 \leq z<\infty,
\end{aligned}
$$

which is a gamma distribution. Therefore,

$$
\lambda_{t+u \mid h_{t}}=\int_{0}^{\infty} \lambda_{t+u \mid h_{t}, z} \pi_{t}(z) \mathrm{d} z=\frac{(n+1) \lambda}{\theta+\lambda t} .
$$

Now the other measures are given by

$$
\begin{aligned}
& \mathbb{P}\left[T_{t} \geq x \mid \mathscr{H}_{t}=h_{t}\right]=\int_{0}^{\infty} \exp \{-z \lambda x\} \pi_{t}(z) \mathrm{d} z=\frac{(\theta+\lambda t)^{n+1}}{(\theta+\lambda t+\lambda x)^{n+1}}, \quad x \geq 0, \\
& \mathbb{P}\left[N_{t}(u)=k \mid \mathscr{H}_{t}=h_{t}\right] \\
& \quad=\int_{0}^{\infty} \frac{(z \lambda u)^{k}}{k !} \exp \{-z \lambda u\} \pi_{t}(z) \mathrm{d} z \\
& \quad=\left(\begin{array}{c}
n+k \\
k
\end{array}\right)\left(\frac{\lambda u}{\theta+\lambda t+\lambda u}\right)^{k}\left(\frac{\theta+\lambda t}{\theta+\lambda t+\lambda u}\right)^{n+1}, \quad k=0,1,2, \ldots,
\end{aligned}
$$

and

$$
\mathbb{E}\left[N_{t}(u) \mid \mathscr{H}_{t}=h_{t}\right]=\int_{0}^{\infty} z \lambda u \pi_{t}(z) \mathrm{d} z=\frac{(n+1) \lambda u}{\theta+\lambda t} .
$$

Case II: suppose that $h_{t}=\{N(t-)=0\}$. In this case,

$$
f_{A(0)}(x, z)=z \lambda \exp \{-z \lambda x\}, \quad 0 \leq x<s,
$$

and

$$
\xi(t, s)=\frac{\theta}{\theta+\lambda t} .
$$

Furthermore, $\lambda_{t+u \mid h_{t}, z, a}$ in (9) is given by

$$
\lambda_{t+u \mid h_{t}, z, a}=z \lambda .
$$

Therefore,

$$
\lambda_{t+u \mid h_{t}}=\frac{\theta+\lambda t}{\theta} \int_{0}^{\infty} z \lambda \exp \{-\lambda t z\} \theta \exp \{-\theta z\} \mathrm{d} z=\frac{\lambda}{\theta+\lambda t} .
$$

The other measures in this case are given by

$$
\begin{aligned}
\mathbb{P}\left[T_{t} \geq x \mid \mathscr{H}_{t}=h_{t}\right] & =\frac{\theta+\lambda t}{\theta} \int_{0}^{\infty} \exp \{-z \lambda x\} \exp \{-\lambda t z\} \theta \exp \{-\theta z\} \mathrm{d} z \\
& =\frac{\theta+\lambda t}{\theta+\lambda t+\lambda x}, \quad x \geq 0, \\
\mathbb{P}\left[N_{t}(u)=k \mid \mathscr{H}_{t}=h_{t}\right] & =\frac{\theta+\lambda t}{\theta} \int_{0}^{\infty} \frac{(z \lambda u)^{k}}{k !} \exp \{-z \lambda u\} \exp \{-\lambda t z\} \theta \exp \{-\theta z\} \mathrm{d} z \\
& =\left(\frac{\lambda u}{\theta+\lambda t+\lambda u}\right)^{k} \frac{\theta+\lambda t}{\theta+\lambda t+\lambda u}, \quad k=0,1,2, \ldots,
\end{aligned}
$$


and

$$
\mathbb{E}\left[N_{t}(u) \mid \mathscr{H}_{t}=h_{t}\right]=\frac{\theta+\lambda t}{\theta} \int_{0}^{\infty} z \lambda u \exp \{-\lambda t z\} \theta \exp \{-\theta z\} \mathrm{d} z=\frac{\lambda u}{\theta+\lambda t}
$$

\section{Point process for minimal repair}

In this section we consider the point process for minimal repairs in heterogeneous populations. In Cha and Finkelstein (2011), a simple consideration for the stochastic intensity of minimally repaired items was made, where the main interest is in obtaining the ordinary stochastic intensity and its comparison. As the main concern of this current paper is the stochastic predictions, we now further extend the discussion and deal with the extended issues under a more general setting. In Subsection 3.1, general results will be derived and an application of the results to reliability will be considered in Subsection 3.2.

\subsection{Main result}

In order to define the minimal repair processes to be considered in this section precisely, as in Section 2, we start our discussion with an explanatory example, which is a variant of Example 1.

Example 3. (Example 1 continued.) As in Example 1, assume that at time $t=0$ one item is chosen from a substock which was randomly selected in accordance with a discrete distribution $\pi(i), i=1,2, \ldots, k$. This item is put into operation and the operating item is now 'minimally' repaired on its failures. Here, by 'minimal repair' we mean that the state of the item after the repair is restored to the 'as-bad-as-old' condition. For instance, if the initial item is chosen from substock $i$ and it has failed at time $x$, then this type of repair implies that the CDF of the repaired item is given by

$$
1-\frac{\bar{F}(x+t, i)}{\bar{F}(x, i)} .
$$

Thus, this type of repair restores our system to the state (defined by the CDF) it had prior to the failure. In practice, the minimal repair can be performed using the following 'operations'.

- The failed system is replaced by a statistically identical system (with the same CDF) 'from the same substock' that was operating for the same time but did not fail.

- The system consists of 'a large number' of elementary components and, therefore, the replacement of only the failed component by an as-good-as-new component, essentially 'does not affect the overall reliability characteristic of the system' and, accordingly, it does not change the system's failure rate. Another example of this 'physical operation' is when a realized defect (fault) is corrected upon failure, whereas the number of possible inherent defects in the system is very large. The minimal repair defined above is considered a reasonable approximation in this case.

Note that we consider a minimal repair process with the frailty variable $Z=z$ of the initial item 'preserved'. Therefore, this type of minimal repair can also be considered as the information-based (or physical) minimal repair, which brings our item back to the state (defined by the relevant information) it had just prior to the failure (see Aven and Jensen (1999), (2000) and Finkelstein (1992), (2004)). Obviously, this point process is not an NHPP as it does not possess the independent increments. However, it is clear that the point process given $Z=z$ follows an NHPP. Therefore, this point process can be called a conditional nonhomogeneous 
Poisson process (cf. Freedman (1962) and Kingman (1964)). The stochastic prediction of this point process will be the main focus of this section.

Now we are ready to formally describe our problem. As in the perfect repair process model, let us suppose that the point process is started at time $t=-s$, but that the process is not observed before time $t=0$. We start the observation on the point process at time $t=0$ and observe it in the time interval $[0, t)$. Very often in practice, the age of the item under operation or under study may be unknown (e.g. operations of used items with unknown age or observation of lifetimes with left censoring). This makes it natural to model it by an RV $S$ and to introduce a random initial starting point. Suppose that $S$ is an absolutely continuous random variable with corresponding CDF and PDF $G(s)$ and $g(s)$, respectively, representing the information/uncertainty about the starting point. In this situation, the process history updates not only the information on the frailty variable, but the information on the unknown starting point as well. Also, it is natural to assume that $Z$ and $S$ are stochastically independent.

We now describe the stochastic measures for the future process $\left\{N_{t}(u), u \geq 0\right\}$ in the following theorem.

Theorem 2. Under the minimal repair process, given $\mathscr{H}_{t}$, the stochastic measures for the process $\left\{N_{t}(u), u \geq 0\right\}$ are given as

(i) $\lambda_{t+u \mid h_{t}}=\int_{0}^{\infty} \int_{0}^{\infty} \lambda(s+t+u, z) \psi_{t}(s, z) \mathrm{d} s \mathrm{~d} z$,

(ii) $\mathbb{P}\left[T_{t} \geq x \mid \mathscr{H}_{t}=h_{t}\right]=\int_{0}^{\infty} \int_{0}^{\infty} \exp \left\{-\int_{0}^{x} \lambda(s+t+v, z) \mathrm{d} v\right\} \psi_{t}(s, z) \mathrm{d} s \mathrm{~d} z$,

(iii) $\mathbb{P}\left[N_{t}(u)=k \mid \mathscr{H}_{t}=h_{t}\right]$

$$
\begin{aligned}
=\int_{0}^{\infty} \int_{0}^{\infty} & \frac{\left(\int_{0}^{u} \lambda(s+t+v, z) \mathrm{d} v\right)^{k}}{k !} \exp \left\{-\int_{0}^{u} \lambda(s+t+v, z) \mathrm{d} v\right\} \\
& \times \psi_{t}(s, z) \mathrm{d} s \mathrm{~d} z,
\end{aligned}
$$

(iv) $\mathbb{E}\left[N_{t}(u) \mid \mathscr{H}_{t}=h_{t}\right]=\int_{0}^{\infty} \int_{0}^{\infty}\left[\int_{0}^{u} \lambda(s+t+v, z) \mathrm{d} v\right] \psi_{t}(s, z) \mathrm{d} s \mathrm{~d} z$,

where $\psi_{t}(s, z)$ is the conditional joint PDF of $(S, Z)$ given $\mathscr{H}_{t}=h_{t}$, i.e.

$$
\psi_{t}(s, z)=\frac{\prod_{i=1}^{n} \lambda\left(s+\sum_{j=1}^{i} t_{j}, z\right) \exp \left\{-\int_{0}^{t} \lambda(s+w, z) \mathrm{d} w\right\} g(s) \pi(z)}{\int_{0}^{\infty} \int_{0}^{\infty} \prod_{i=1}^{n} \lambda\left(r+\sum_{j=1}^{i} t_{j}, v\right) \exp \left\{-\int_{0}^{t} \lambda(r+w, v) \mathrm{d} w\right\} g(r) \pi(v) \mathrm{d} r \mathrm{~d} v}
$$

for $0 \leq s, z<\infty$, and $\prod_{i=1}^{n}(\cdot) \equiv 1$ for $n=0$.

Proof. It is now clear that

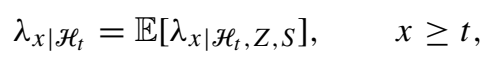

where the expectation is with respect to the joint 'conditional distribution' of $\left(S, Z \mid \mathscr{H}_{t}\right)$, and

$$
\lambda_{x \mid \mathcal{H}_{t}, Z, S} \equiv \lim _{\Delta t \rightarrow 0} \frac{\mathbb{P}\left[N(x, x+\Delta t)=1 \mid \mathscr{H}_{t}, Z, S\right]}{\Delta t}, \quad x \geq t .
$$

Again, the corresponding limit and the notation $\lambda_{x \mid h_{t}, z, s}$ are defined as in the proof of case I of Theorem 1. Then

$$
\lambda_{t+u \mid h_{t}, z, s}=\lim _{\Delta t \rightarrow 0} \frac{\mathbb{P}\left[N(t+u, t+u+\Delta t)=1 \mid \mathscr{H}_{t}=h_{t}, Z=z, S=s\right]}{\Delta t} .
$$


Note that, given $\left(\mathscr{H}_{t}=h_{t}, Z=z, S=s\right)$, owing to the property of the information-based minimal repair defined before (i.e. each failure does not change the age or the frailty variable of the item in use), the age and the frailty variable of the item in operation at time $t+u$ is given by $s+t+u$ and $z$, respectively. Furthermore, owing to the property of the informationbased minimal repair, the repair does not change the shape of the corresponding failure rate. Accordingly, we have $\lambda_{t+u \mid h_{t}, z, s}=\lambda(s+t+u, z)$. Therefore,

$$
\lambda_{t+u \mid \mathscr{H}_{t}, Z, S}=\lambda(S+t+u, Z), \quad u \geq 0 .
$$

Note that the conditional distribution of the history $\mathscr{H}_{t}=h_{t}$ given $(S=s, Z=z)$ is

$$
\begin{aligned}
& \lambda\left(s+t_{1}, z\right) \exp \left\{-\int_{0}^{t_{1}} \lambda(s+w, z) \mathrm{d} w\right\} \lambda\left(s+t_{1}+t_{2}, z\right) \exp \left\{-\int_{t_{1}}^{t_{1}+t_{2}} \lambda(s+w, z) \mathrm{d} w\right\} \\
& \times \cdots \times \lambda\left(s+\sum_{j=1}^{n} t_{j}, z\right) \exp \left\{-\int_{\sum_{j=1}^{n-1} t_{j}}^{\sum_{j=1}^{n} t_{j}} \lambda(s+w, z) \mathrm{d} w\right\} \\
& \times \exp \left\{-\int_{\sum_{j=1}^{n} t_{j}}^{t} \lambda(s+w, z) \mathrm{d} w\right\} \\
&= \prod_{i=1}^{n} \lambda\left(s+\sum_{j=1}^{i} t_{j}, z\right) \exp \left\{-\int_{0}^{t} \lambda(s+w, z) \mathrm{d} w\right\},
\end{aligned}
$$

where $\prod_{i=1}^{n}(\cdot) \equiv 1$ for $n=0$. Therefore, the conditional joint PDF of $(S, Z)$ given $\mathscr{H}_{t}=h_{t}$, $\psi_{t}(s, z)$, is given by (11). Then, in accordance with (12),

$$
\lambda_{t+u \mid h_{t}}=\int_{0}^{\infty} \int_{0}^{\infty} \lambda(s+t+u, z) \psi_{t}(s, z) \mathrm{d} s \mathrm{~d} z .
$$

Results (ii), (iii), and (iv) can also be obtained following a similar procedure.

\subsection{Application}

In the previous section we considered a continuous mixing model. In this subsection we consider a discrete mixing model, which can be used to illustrate how the formulation can be modified to a simpler case when necessary. Suppose that the population is composed of the mixture of two exponential distributions with parameters (failure rates) $\lambda_{1}$ and $\lambda_{2}, \lambda_{1}<\lambda_{2}$. The composition of our mixed population is as follows: the proportion of the items with $\lambda_{1}$ (to be called 'strong' items) is $\pi$, whereas the proportion of the items with $\lambda_{2}$ (to be called 'weak' items) is $1-\pi$. Then the frailty variable $Z$ in this case has a discrete probability distribution

$$
\pi(z)= \begin{cases}\pi, & z=1, \\ 1-\pi, & z=2,\end{cases}
$$

where $z=i, i=1,2$, corresponds to 'strong' and 'weak' subpopulations, respectively.

Suppose that a new item is randomly chosen from the mixed population and that it is tested during $[0, t)$ before a field operation. During this test period, the item is repaired following the minimal repair process described in the previous subsection. Then, based on the information obtained during the period, $\mathscr{H}_{t}$, the item is either eliminated or put into a field operation. Observe that this kind of elimination procedure can be considered as a kind of burn-in procedure (see 
Jensen and Petersen (1982), Mi (1994), and Cha (2000)). However, in conventional burn-in procedures, items are subjected to a period of simulated use prior to actual operation and those which failed during this period are scrapped and discarded. In this conventional case, the information which is used for the burn-in procedure is whether the items have survived the burn-in period or not, the failure times for failed items are not used.

On the other hand, in the elimination procedure described above, the items that have gone through the procedure possess different information about the reliability characteristics, which can then be used for establishing the elimination procedure. Based on this reasoning, the following elimination procedure can be considered. Let $\tau$ be the mission time in the field operation. For a given integer $k^{*}$, define a set of histories

$$
\mathbb{H}_{\mathrm{p}} \equiv\left\{h_{t} \mid \mathbb{P}\left[N_{t}(\tau) \leq k^{*} \mid \mathscr{H}_{t}=h_{t}\right] \geq \alpha\right\},
$$

where $\alpha, 0<\alpha<1$, is a predetermined threshold level. Then, given the history in $[0, t)$ of a tested item, $\mathscr{H}_{t}^{\prime}=h_{t}^{\prime}$, the elimination policy can now be defined as

(i) if $h_{t}^{\prime} \in \mathbb{H}_{\mathrm{p}}$ then the item is put into a field operation;

(ii) otherwise, it is eliminated.

That is, by the above procedure, only the items with probabilities (that the number of failures during the mission does not exceed $k^{*}$ ) higher than $\alpha$ (the threshold level) pass the elimination procedure and are put into field operations.

Observe that, as the starting point in this case is given by $S \equiv 0$ (a degenerate distribution), from the proof of Theorem 2

$$
\psi_{t}(z) \equiv \mathbb{P}\left[Z=z \mid \mathscr{H}_{t}=h_{t}\right]=\frac{\lambda_{z}^{n} \exp \left\{-\lambda_{z} t\right\} \pi(z)}{\sum_{i=1}^{2} \lambda_{i}^{n} \exp \left\{-\lambda_{i} t\right\} \pi(i)}, \quad z=1,2,
$$

which does not depend on the failure points, but depends only on the number of failures during the period. Therefore, as far as only the corresponding stochastic predictions are involved, the operational history in this case is completely characterized by $N(t-)$. Then the elimination procedure described above can be restated in terms of $N(t-)$ as follows. Define a set of integers

$$
\mathbb{N}_{\mathrm{p}} \equiv\left\{n \mid \mathbb{P}\left[N_{t}(\tau) \leq k^{*} \mid N(t-)=n\right] \geq \alpha\right\} .
$$

Then, given the number of failures in $[0, t)$ of a tested item, $N^{\prime}(t-)=n^{\prime}$, the elimination policy can now be described as

(i) if $n^{\prime} \in \mathbb{N}_{\mathrm{p}}$ then the item is put into a field operation;

(ii) otherwise, it is eliminated.

Observe that

$$
\mathbb{P}\left[N_{t}(\tau) \leq k^{*} \mid N(t-)=n\right]=\sum_{z=1}^{2}\left(\sum_{k=0}^{k^{*}} \frac{\left(\lambda_{z} \tau\right)^{k} \exp \left\{-\lambda_{z} \tau\right\}}{k !}\right) \frac{\lambda_{z}^{n} \exp \left\{-\lambda_{z} t\right\} \pi(z)}{\sum_{i=1}^{2} \lambda_{i}^{n} \exp \left\{-\lambda_{i} t\right\} \pi(i)} .
$$

It is clear that $\mathbb{P}\left[N_{t}(\tau) \leq k^{*} \mid N(t-)=n\right]$ is strictly decreasing in $n$ and that it has its maximum at $n=0$. Therefore, if $\alpha$ satisfies

$$
0<\alpha \leq \sum_{z=1}^{2}\left(\sum_{k=0}^{k^{*}} \frac{\left(\lambda_{z} \tau\right)^{k} \exp \left\{-\lambda_{z} \tau\right\}}{k !}\right) \frac{\exp \left\{-\lambda_{z} t\right\} \pi(z)}{\sum_{i=1}^{2} \exp \left\{-\lambda_{i} t\right\} \pi(i)}
$$


then the corresponding elimination policy can be established. Suppose that there are missions of different importance in the field operations. Then, as the items that have passed the elimination procedure possess information about the quality (or reliability) level, items with a higher reliability level (i.e. items with smaller $N^{\prime}(t-)$ ) could be used for more important missions.

Moreover, the 'average stochastic measures' for items that have passed the elimination procedure can also be obtained. Note that the set $\mathbb{N}_{p}$ in (14) is given by

$$
\mathbb{N}_{\mathrm{p}}=\left\{0,1, \ldots, n^{*}\right\}
$$

where $n^{*}$ is the maximum integer value satisfying

$$
\mathbb{P}\left[N_{t}(\tau) \leq k^{*} \mid N(t-)=n\right] \geq \alpha .
$$

Observe that, just after the elimination procedure, the initial frailty distribution for items that have passed the procedure is given by

$$
\begin{aligned}
\pi^{*}\left(z \mid n^{*}\right) & \equiv \mathbb{P}\left[Z=z \mid N(t-) \leq n^{*}\right] \\
& =\frac{\left[\sum_{k=0}^{n^{*}}\left(\lambda_{z} t\right)^{k} \exp \left\{-\lambda_{z} t\right\} / k !\right] \pi(z)}{\sum_{i=1}^{2}\left[\sum_{k=0}^{n^{*}}\left(\lambda_{i} t\right)^{k} \exp \left\{-\lambda_{i} t\right\} / k !\right] \pi(i)}, \quad z=1,2 .
\end{aligned}
$$

Then the average stochastic measures for items that have passed the elimination procedure can be obtained by replacing $\psi_{t}(z)$ in (13) with $\pi^{*}\left(z \mid n^{*}\right)$ in the corresponding derivations of measures in Theorem 2.

\section{Concluding remarks}

In this paper we have considered the failure processes of repairable items from heterogeneous populations. Two types of repair processes, perfect and minimal repair processes, have been considered. The point processes that have been considered are not pure renewal processes/NHPPs, but conditional renewal processes/NHPPs, respectively. Based on the failure/repair history, the stochastic predictions of the future processes were studied.

In Section 3, an application of the results to reliability was considered. Burn-in is an important issue in reliability and various methodologies have been proposed. However, there has been little literature on the burn-in procedure, which utilizes the failure/repair history of the item during the process. The application has been illustrated assuming exponential distributions and the suggested model could hint at general research in this direction. Based on this initial setup, a general study of the topic is being performed by the author. Similar approaches could also be applied to the study of maintenance policies for repairable items from heterogeneous populations. In this case, it is clear that the maintenance policy should depend on the subpopulation from which the item is selected and the corresponding failure rate. Further studies in this field are needed, with the formulation and discussions in this paper providing a useful starting point.

In this paper, for a convenient formulation, the problem has been described in the context of reliability modeling. However, the obtained results could be applied to various relevant areas. For example, similar kinds of lack of information due to population heterogeneity considered in this paper may also frequently happen in queueing analysis, where the arrival process of customers may exhibit a heterogeneous pattern (see Yechiali and Naor (1971), Niu (1980), and Böhm and Mohanty (1994)). In this case, a precise prediction of the arrival process is practically a very important issue (see also Cha and Lee (2011)). The results could also be 
applied to life insurance when the insured are from heterogeneous populations, where the recurrent occurrences of illness may correspond to the recurrent events in the point processes. On the other hand, the predictions of natural disasters such as earthquakes and river floods are of practical importance (see Molchan (1990), (1997) and Werner et al. (2011)), where renewal processes are frequently adopted. The obtained results could also be applied to these areas.

\section{Acknowledgements}

The author is very grateful to the anonymous referee for helpful comments and a careful reading of this paper, which have considerably clarified its presentation. This work was supported by a National Research Foundation of Korea (NRF) grant funded by the Korean government (MEST) (no. 2011-0017338). The author would also like to thank Mrs Sul Ja Choi for helpful and pleasant discussions which led to a practical formulation of the problem given in the paper.

\section{References}

Aven, T. And Jensen, U. (1999). Stochastic Models in Reliability. Springer, New York.

Aven, T. And Jensen, U. (2000). A general minimal repair model. J. Appl. Prob. 37, 187-197.

BADía, F. G. (2011). Hazard rate properties of a general counting process stopped at an independent random time. J. Appl. Prob. 48, 56-67.

Badía, F. G. AND SANGgüEssa, C. (2008). Preservation of reliability classes under mixtures of renewal processes. Prob. Eng. Inf. Sci. 22, 1-17.

Böнm, W. and Mohanty, S. G. (1994). Transient analysis of queues with heterogeneous arrivals. Queueing Systems 18, 27-45.

Cha, J. H. (2000). On a better burn-in procedure. J. Appl. Prob. 37, 1099-1103.

Cha, J. H. AND Finkelstein, M. (2011). Stochastic intensity for minimal repairs in heterogeneous populations. J. Appl. Prob. 48, 868-876.

ChA, J. H. AND LeE, E. Y. (2011). A stochastic breakdown model for an unreliable web server system and optimal admission control policy. J. Appl. Prob. 48, 453-466.

Cox, D. R. (1962). Renewal Theory. Methuen, London.

Esary, J. D., Marshall, A. W. and Proschan, F. (1973). Shock models and wear processes. Ann. Prob. 1, $627-649$.

Finkelstein, M. S. (1992). Some notes on two types of minimal repair. Adv. Appl. Prob. 24, 226-228.

Finkelstein, M. S. (2004). Minimal repair in heterogeneous populations. J. Appl. Prob. 41, 281-286.

Finkelstein, M. (2008). Failure Rate Modelling for Reliability and Risk. Springer, London.

FinkelsteIn, M. (2009). Understanding the shape of the mixture failure rate (with engineering and demographic applications). Appl. Stoch. Models Business Industry 25, 643-663.

Freedman, D. A. (1962). Poisson processes with random arrival rate. Ann. Math. Statist. 33, 924-929.

Grandell, J. (1997). Mixed Poisson Processes. Chapman and Hall, London.

Kingman, J. F. C. (1964). On the doubly stochastic Poisson processes. Proc. Camb. Phil. Soc. 60, 923-930.

Mi, J. (1994). Burn-in and maintenance policies. Adv. Appl. Prob. 26, 207-221.

Molchan, G. M. (1990). Strategies in strong earthquake prediction. Phys. Earth Planetary Interiors 61, 84-98.

Molchan, G. M. (1997). Earthquake prediction as a decision-making problem. Pure Appl. Geophys. 149, $233-247$.

NiU, S. C. (1980). A single server queueing loss model with heterogeneous arrival and service. Operat. Res. 28, 584-593.

Rausand, M. and Høyland, A. (2004). System Reliability Theory: Models, Statistical Methods, and Applications, 2nd edn. John Wiley, Hoboken, NJ.

Werner, M. J., IdE, K. AND Sornette, D. (2011). Earthquake forecasting based on data assimilation: sequential Monte Carlo methods for renewal point processes. Nonlinear Process. Geophys. 18, 49-70.

Yechiali, U. and NaOR, P. (1971). Queuing problems with heterogeneous arrivals and service. Operat. Res. 19, $722-734$. 